\title{
Sur les crues survenues en France de 1951 à 1956
}

\section{Concerning floods that have occured in France between 1951 and 1956}

\author{
PAR M. PARDÉ
}

PROFESSEUR A L'INSTITUT DE GÉOGRAPHIE ALPINE ET A L'INSTITUT POLYTECHNIQUE DE GRENOBLE

\begin{abstract}
Le conférencier prie les personnes qui désireraient obtenir sur ces phénomènes signalés par lui, des données imprimées précises, de lire sous le même titre, une communication qu'il a présentée au Symposium Darcy de Dijon (20-26 septembre 1956) - et qui se trouve contenue - pp. 207-221, dans le tome $I I I$ (Les Crues, publication $n^{\circ} 42$ de l'Association Internationale d'Hydrologie Scientifique), des publications relatives audit Corigrès.
\end{abstract}

Le bref résumé qui suit expose les principales inondations survenues en France dans la période considérée.

\begin{abstract}
The lecturer invites those who are interested in obtaining precise printed information on the phenomena described by him, to consult a paper given by him on the same subject before the Darcy Symposium at Dijon (20-26 september, 1956) and which will be found on pages 207-221 of Volume III (Floods, publication $N^{\circ} 42$ of the International Association of Scientific Hydrology) of the publication relative to this Congress.
\end{abstract}

The brief summary which follows describes the more important floods that have occured in France during the period considered.
La médiocrité sensible de l'hydraulicité moyenne, de 1953 à 1955 en France, a dû plus que compenser les excédents des detux années précédentes. Cela n'a pas empêché les cinq ans 1951-1955 de connaître un nombre anormal de grandes crues dans notre pays.

La Garonne et l'Adour ont éprouvé une crue quasi grandiose de type océanique pyrénéen en février 1952. A Dax-sur-l'Adour, le niveau atteint n'a été dépassé que par celui d'avril 1770. Sur la Garonne moyenne et inférieure, qui souvent déborde très largement, on ne connaît que trois crues supérieures à celle de février 1952. En janvier 1955, le phénomène n'eut de gravité qu'en aval du confluent avec le Lot, et il fut sensiblement inférieur au précédent. Mais cette crue, due à plusieurs averses rapprochées en une dizaine de jours, se distingue par une extra- ordinaire durée des très hautes eaux, événement rare pour la Garonne.

Une crue à moitié contemporaine, mais un peu plus précoce, de la Seine a causé les plus vives alarmes. Une averse de plus vers le 19 ou le 20 l'aurait rendue aussi terrible que l'inondation mémorable de janvier 1910. Et la Seine supérieure à Bray n'a jamais été plus haute de mémoire d'homme.

La longue tenue des hautes eaux a rendu sur les tributaires la même intumescence tout à fait imposante sur la Saône inférieure. Et le Rhône supérieur, l'Isère, etc., s'étant gonflés plusieurs fois dans le même temps, cette crue, à Avignon et à Beaucaire, égala des crues méditerranéennes déjà sérieuses, fait presque sans précédent. Mais en novembre 1951, deux phénomènes méditerranéens, dont l'un accompagna le désastre 
causé le long du Pô, comportèrent sur le cours inférieur du Rhône des cotes imposantes, et même remarquables lors de la deuxième poussée, presque égale à la crue de novembre 1935 (mais très inférieure aux records de 1840 et 1856).

La Dordogne et la Loire inférieure eurent de grandes crues non exceptionnelles en décembre 1952. La Saône déborda très largement en avril 1952 , et chose plus frappante, vu la date inhabituelle en juin 1953 (plus grande crue connue de la Loire). Enfin, l'Orb à Béziers eut en décembre 1953 une crue record.

Enfin, M. Pardé a élaboré sur les mêmes événements un mémoire triple de celui dont nous faisons mention plus haut. En attendant la publication éventuelle de ce texte, il peut en prêter un exemplaire dactylographié aux sociétaires désireux de le consulter.

COMMENTAIRE

de M. le Président KocH.

M. le Président remercie M. Pardé de son exposé extrêmement circonstancié sur un certain nombre de crues dont il a fait ressortir les éléments caractéristiques : soit des éléments permanents comme ceux du type océanique, du type méditerranćen général, soit les variations qui s'y greffent (orientations différentes, interaction des pluies atlantiques et méditerranéennes) et en particuliex l'orientation un peu spéciale, très nuancée, qui amène des crues sur le bassin de la Garonne et de l'Adour.

M. PARDÉ a d'ailleurs fait observer très justement que ce n'est pas toujours l'intensité des dépressions au voisinage de la surface du sol qui commande les avalaisons de crues et qu'il peut intervenir d'autres phénomènes à plus ou moins haute altitude; la recherche plus étendue des pressions atmosphériques et des anticyclones serait sans doute susceptible de donner à cet égard des renseignements complémentaires dont on ne saurait sousestimer l'intérêt.

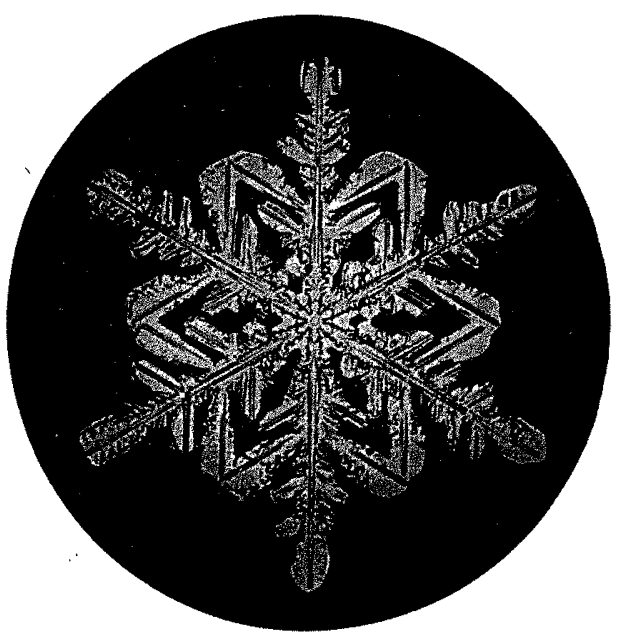

